\title{
Implantes dentários curtos na implantodontia moderna: revisão sistematizada
}

\author{
Valthierre Nunes de LIMA \\ Guilherme André Del'Arco RAMIRES \\ Júlio Cesar Monteiro BARBOSA \\ Tárik Ocon Braga POLO \\ Leonardo Perez FAVERANI \\ Ana Paula Farnezi BASSI
}

Short implants dental in modern implantology: systematized review

Implantes dentales curtos en la implantodontia moderna: revisión sistematizada

\begin{abstract}
Resumo
Introdução: Os implantes curtos têm ganhado espaço na implantodontia moderna devido se tratar de uma técnica que apresenta baixa morbidade, sem necessidade de cirurgias de enxertos prévias e menor custo para o paciente, quando comparada a outras técnicas. Objetivo: Dessa forma, foi realizada uma revisão da literatura atual sobre as vantagens e indicações desta técnica comparando-a as técnicas convencionais. Materiais e métodos: A pesquisa bibliográfica foi realizada usando MEDLINE via PubMed, utilizando termos e outros termos livres dos Cabeçalhos de Assunto Médicos (MeSH) para pesquisar. Abrangendo artigos publicados em inglês, dos ultimos 18 anos. Resultados: Foram selecionados 56 referências, das quais 26 foram consideradas inadequadas. Resultando no total de 30 artigos considerados adequados aos critérios de seleção. Os artigos selecionados apresentaram em alguns pontos divergência que ainda necessitam de respostas baseadas em evidencias. Conclusão: Conclui-se com esta revisão que os implantes curtos são uma boa alternativa quando não se tem altura óssea suficiente para instalação de implantes convencionais, e que apresenta vantagens e indicações restritas no universo da implantodontia com resultados similares quando comparados aos implantes de tamanhos convencionais. Porém, apresentam resultados a curto prazo o que sugere a realização de estudos com longo tempo de acompanhamento que busquem responder sobre a qualidade da técnica nos diversos desafios encontrados na implantodontia.
\end{abstract}

Programa de Pós-Graduação em Odontologia, Faculdade de Odontologia de Araçatuba, Univ Estadual Paulista, UNESP 16015.050 Araçatuba-SP, Brasil

Descritores: Implantes Dentários; Reabsorção Óssea; Reabilitação Bucal.

\begin{abstract}
Introduction: The short implants have an open space in modern implantology due to a technique that presents low morbidity, without previous grafting surgeries and lower cost for the patient, when compared to other techniques. Objective: Thus, a review of current literature on the advantages and indications of technique was carried out comparing conventional techniques. Materials and methods: Bibliographic research was conducted using MEDLINE via PubMed, using terms and other free Medical Subject Headings (MeSH) terms to search for. Covering articles published in English, from the last 18 years. Results: We selected 56 references, of which 26 were considered inadequate. Resulting in a total of 30 articles considered adequate to the selection criteria. The selected articles presented in some points divergence that still need evidence based answers. Conclusion: It was concluded with this review that short implants are a good alternative when there is not enough height for the installation of conventional implants, and that they have advantages and restricted indications no universe of the implantology with similar results when compared to the implants of conventional sizes. However, results of a short term and that suggests a course of studies with a time of accompaniment that seek answers on a quality of the technique in the diverse challenges found in the implantology.
\end{abstract}

Descriptors: Dental Implants; Bone Resorption; Mouth Rehabilitation.

\section{Resumen}

Introducción: Los implantes cortos han ganado espacio en la implantodoncia moderna debido a que se trata de una técnica que presenta baja morbilidad, sin necesidad de cirugías de injertos previos y menor costo para el paciente, en comparación con otras técnicas. Objetivo: De esta forma, se realizó una revisión de la literatura actual sobre las ventajas e indicaciones de esta técnica comparándola con las técnicas convencionales. Materiales y métodos: La investigación bibliográfica fue realizada usando MEDLINE vía PubMed, utilizando términos y otros términos libres de los Cabeceros de Asunto Médicos (MeSH) para investigar. Abriendo artículos publicados en inglés, de los últimos 18 años. Resultados: Se seleccionaron 56 referencias, de las cuales 26 fueron consideradas inadecuadas. Resultando en el total de 30 artículos considerados adecuados a los criterios de selección. Los artículos seleccionados presentaron en algunos puntos divergencia que aún necesitan respuestas basadas en evidencias. Conclusión: Se concluye con esta revisión que los implantes cortos son una buena alternativa cuando no se tiene suficiente altura ósea para instalación de implantes convencionales y que presenta ventajas e indicaciones restringidas en el universo de la implantodoncia con resultados similares en comparación con los implantes de tamaños convencionales. Sin embargo, presentan resultados a corto plazo lo que sugiere la realización de estudios con largo tiempo de acompañamiento que busquen responder sobre la calidad de la técnica en los diversos desafíos encontrados en la implantodoncia.

Descriptores: Implantes Dentales; Reasorción Ósea; Rehabilitación Bucal.

\section{INTRODUÇÃO}

A implantodontia surgiu como uma ferramenta promissora para solucionar os problemas ocasionados pela perda dentaria. Nos últimos 50 anos os implantes osseointegráveis e suas técnicas de instalações passaram de um tratamento experimental para uma opção com taxa de sucesso acima de $95 \%$. As características funcionais e biológicas desta modalidade de tratamento têm ganhado cada vez mais a confiança e aceitação dos pacientes portadores de edentulismo total ou parcial devido os resultados satisfatórios a longo prazo ${ }^{1}$.
Considerando o sucesso destes implantes, sua indicação tem se tornado uma prática diária nos consultórios e clínicas odontológicas. Desta forma, os desafios encontrados em cada paciente impulsionam a evolução da implantodontia através de meios alternativos para a instalação dos implantes quando não se existe quantidade ou qualidade óssea suficiente para o sucesso da osseointegração ${ }^{2}$.

Neste contexto, tanto a engenharia genética como a utilização de biomateriais tem crescido no mercado, porém ambas elevam os custos da 
reabilitação. Contudo, existem casos limítrofes que podem ser abordados através de técnicas $\mathrm{e}$ tecnologias menos onerosas ao paciente como a confecção de osteotomias que geram expansões ósseas ou instalação de implantes de menor tamanho.

A principal vantagem de um implante curto é a possibilidade de instalação em regiões com pouca altura óssea evitando assim a entrar na cavidade sinusal ou lesão nervosa e a necessidade de um enxerto ósseo, embora ainda seja necessário um mínimo de 5-6 mm de altura residual do osso ${ }^{3}$.

O objetivo deste trabalho foi realizar uma revisão atual da literatura sobre a reabilitação protética através de implantes curtos.

\section{MATERIAL E MÉTODO}

\section{Estratégia de Busca}

A pesquisa foi realizada usando MEDLINE via PubMed, utilizando termos e outros termos livres dos Cabeçalhos de Assunto Médicos (MeSH) para pesquisar. Foram aplicados os seguintes descritores, para a pesquisa: dental implants AND bone resorption; dental implants AND mouth rehabilitation. Isso abrangeu artigos publicados em inglês, no período entre 1999 e 2016.

Critérios de Seleção

A estratégia de busca foi projetada para identificar artigos relacionados ao tema de implantes curtos que se concentraram nas técnicas cirúgicas adaptadas e convencionais, no sucesso de osseointegração após o carregamento protético e comparação com implantes de comprimento aumentado.

\section{Critérios de Exclusão}

Foram excluídos os artigos que nao apresentavam implantes curtos no tema ou que trouxeessem dados pouco relevantes para este estudo. Desfecho Primário

Nesta revisão buscou-se a taxa de sobrevivência dos implantes em função do tempo de acompanhamento e por meio da comparação entre os tipos de implantes: curtos versus de comprimento convencional.

\section{RESULTADOS}

A estratégia de pesquisa identificou 703 referências com o descritor dental implants AND mouth rehabilitation, e 5106 com o descritor dental implants AND bone resorption.

Após a pesquisa, 5753 artigos foram considerados inadequados para esta revisão, devido ao fato de que o título e / ou abstract não se enquadraram aos critérios de inclusão. Foram lidos os textos completos de 56 artigos, dos quais 26 também não se enquadraram nos critérios de seleção, e foram excluídos. Um total de 30 artigos foram utilizados para realização da presente revisão.

\section{DISCUSSÃO}

Quando considerada a biomecânica dos implantes dentários, os implantes curtos apresentam indicações para qualquer região dos maxilares que apresentem escassez de altura óssea, porem a biomecânica e o comportamento biológico de cada região são distintos. As regiões posteriores dos maxilares são as que apresentam maiores indicações ${ }^{4}$.

Considerando o diâmetro do implante estudos demonstram que ele é mais eficiente que o comprimento na dissipação de tensões ${ }^{5}$. Um comprimento aumentado, portanto, só iria melhorar a estabilidade primária. A análise de elementos finitos demonstrou que o comprimento do implante pode não ser $o$ principal fator que influencia a transferência de cargas oclusais para a interface ossoimplante $^{6}$. Este conceito tem sido bastante aceito e vantajoso quando se relaciona aos implantes curtos devido à sua capacidade de reduzir o pico de tensões na região de cortical óssea ${ }^{7}$.

A qualidade óssea também é um fator primário para o sucesso de implantes curtos. Ossos tipo III e IV mostram mais falhas, independentemente do tratamento de superfície do implante. A combinação de comprimento curto do implante e má qualidade óssea reduz a estabilidade do implante durante a instalação do implante e o período de cicatrização ${ }^{6}$.

O aumento da relação coroa/implante pode atuar como um cantilever vertical levando à perda óssea e falha do implante. Um cantilever magnifica as forças diretamente proporcionais à altura da coroa, criando seis pontos de rotação de potencial diferentes no corpo do implante ${ }^{8}$.

Com relação à configuração ou design, os implantes curtos favorecem a biomecânica. Quanto maior o número de espiras por unidade de comprimento no mesmo plano axial maior será a área da superfície do implante em contato com o osso. Segundo Misch ${ }^{9}$, a forma da rosca quadrada tem uma porcentagem de contato osso-implante maior em comparação com os desenhos de rosca em forma de $\mathrm{V}$ e de contraforte inverso. Além disso, a superfície em micro topografia rugosa aumenta a área de contato osso-implante e acelera a osseointegração em comparação com a lisa. Outro ponto positivo apontado pelo autor é a compensação da relação coroa / implante inadequada ${ }^{9}$.

Dentre os sistemas de conexão, o tipo cone morse induz menor perda óssea marginal em comparação com a conexão do pilar hexagonal externo e também promove o crescimento ósseo sobre o ombro do implante. A conexão do hexágono interno mostra uma distribuição de força mais ampla em comparação com hexágono externo ${ }^{10}$.

Neves et al. ${ }^{11}$ definem como implantes curtos aqueles de comprimento menor que 10 a $11 \mathrm{~mm}$, 
enquanto Renouard e Nisand ${ }^{12}$ consideram nesta categoria apenas os que apresentem $8 \mathrm{~mm}$ ou menos. Revisão sistemática realizada em 2011 por Telleman et al. ${ }^{13}$ concluiu que implantes menores de $10 \mathrm{~mm}$ apresentam boa taxa de sobrevivência com tendência da taxa aumentar de acordo com o tamanho do implante.

Alguns estudos clínicos $^{14,15}$ e revisões sistemáticas ${ }^{12,13}$ publicados na literatura internacional buscaram comparar o sucesso dos implantes curtos comparados aos implantes de tamanhos convencionais. Foi concluído em revisão que os maiores índices de falha para implantes curtos foram relatados em estudos realizados com procedimentos cirúrgicos de rotina, independentemente da qualidade óssea, com implantes de superfície usinada e locais com baixa densidade óssea ${ }^{12}$. Estudos mais recentes realizados com preparação cirúrgica adaptada à densidade óssea e implantes de superfície texturizada relataram taxas de sobrevida comparáveis com aqueles obtidos com implantes de tamanho convencional.

Pacientes tabagistas e/ou diabéticos são portadores dois fortes fatores de risco ligados a sobrevivência dos implantes. Em um estudo clínico comparando a taxa de sobrevivência de implantes em pacientes fumantes e não fumantes e concluiu que $o$ tabagismo intenso não afetou a taxa de sobrevivência, porém diminuiu a velocidade de cicatrização óssea ${ }^{16}$.

Al Amri et $\mathrm{al}^{17}$. concluiram que quanto à presença de diabetes os implantes curtos podem demonstrar estabilidade clínica e radiográfica de uma maneira similar aos implantes longos convencionais em pacientes com e sem diabetes tipo 2 .

Neste contexto, a literatura atual ${ }^{14,15}$ apresenta resultados similares entre implantes curtos e de tamanho convencionais refletindo no questionamento sobre qual a melhor conduta diante de atrofias ósseas severas. O uso de implantes curtos fornece uma opção de tratamento única para aqueles que têm restrições que impeçam os enxertos. Como ainda estamos incertos quanto à qualidade da evidência nesta revisão, é altamente recomendável que sejam realizados estudos clínicos randomizados bem planejados com um acompanhamento de pelo menos 5 anos. Estes devem levar em consideração os possíveis fatores de confusão para esclarecer o efeito dos implantes sobre o sucesso $\mathrm{e}$ as taxas de complicação em pacientes com atrofias ósseas severas.

Importante considerar que diferentes técnicas de aumento ósseo foram introduzidas para superar problema das atrofias maxilares geradas pela reabsorção óssea alveolar associado à pneumatização do seio maxilar. Dentre estes, a elevação do seio maxilar tornou-se o procedimento mais confiável e mais utilizado ${ }^{18}$. Embora empregada com sucesso para regenerar o osso, estudos recentes apontam que esta intervenção cirúrgica aumenta a duração, morbidade e o custo do tratamento ${ }^{19}$. Além disso, podem ocorrer complicações (sinusite pós-operatória, falha parcial ou total do enxerto) após a elevação do seio maxilar ${ }^{20}$.

A colocação de implantes dentários curtos representa uma solução de tratamento alternativa viável e minimamente invasiva para a restauração protética da maxila posterior com quantidade limitada de osso $^{3,21}$. O uso de implantes curtos simplifica a restauração: na verdade, não é necessário elevação do seio maxilar, reduzindo o risco de complicações, tempo e custo de tratamento e, assim, aumentando a aceitação do tratamento pelo paciente $^{21}$.

Uma definição anterior para implante curto é um implante com seu comprimento intraósseo de 8 $\mathrm{mm}^{13}$. Como alternativa à técnica de elevação do seio maxilar, que se provou ser um procedimento cirúrgico previsível, os implantes curtos estão sendo cada vez mais utilizados em maxilas atróficas posteriores $^{22}$.

No entanto, foram relatados resultados contraditórios quando implantes curtos são inseridos em maxilas atróficas posteriores. Um estudo retrospectivo de 4.591 implantes relatou que as taxas de sobrevivência dos implantes colocados nas maxilas foram significativamente menores do que as da mandíbula ( $87 \%$ e $100 \%$, respectivamente $)^{23}$. Em contrapartida, a literatura apresenta taxas de sobrevida aceitáveis (variando de $90,0 \%$ a $98,3 \%$ ) de implantes curtos $(5-7 \mathrm{~mm})$ nas maxilas posteriores ${ }^{24}$.

Em estudo realizado em Shangai com 56 pacientes normossistêmicos, sem patologias locais e não fumantes, com altura óssea remanescente de $6 \mathrm{~mm}$, Zhang et al. ${ }^{4}$ dividiram os pacientes em 3 grupos: grupo 1 com instalação de implantes curtos de $6 \mathrm{~mm}$; grupo 2 com implantes de $8 \mathrm{~mm}$ através da técnica com osteótomos; grupo 3 com implantes de $10 \mathrm{~mm}$ através da técnica de elevação do seio maxilar. Os autores concluíram que todas as técnicas são seguras devido a taxa zero de falha precoce e elevada estabilidade dos implantes ${ }^{4}$.

Morrison et al. ${ }^{25}$ destacam que frequentemente a atrofia óssea das regiões edêntulas posteriores da mandíbula restringe a instalação de implantes dentários, o que apresenta um desafio para os clínicos devido à presença de nervo alveolar inferior (NAI). Segundo os autores, para superar essa limitação em pacientes com atrofia mandibular grave, existem basicamente quatro opções de tratamento, como instalação de implante curto, enxerto ósseo vertical em onlay, distração osteogênica ou lateralização do $\mathrm{NAI}^{25}$.

Técnicas avançadas de aumento ósseo cirúrgico, como o aumento ósseo vertical, são frequentemente realizadas para a posterior ou imediata instalação de implantes com tamanhos 
convencionais $^{26}$. Os procedimentos de aumento ósseo vertical em atrofias severas são tecnicamente sensíveis podendo causar significativa morbidade e complicações como dor intensa e reabsorção elevada do enxerto ${ }^{27}$. Dentro dessas técnicas, a lateralização do nervo é uma das opções de tratamento para a instalação de implantes dentários na região posterior mandibular quando na presença de altura óssea limitada ou nervo alveolar inferior localizado superiormente $^{28}$.

As vantagens da lateralização do nervo podem ser resumidas da seguinte forma: implantes mais longos podem ser instalados no mesmo passo cirúrgico, maior estabilidade primária pode ser obtida devido à fixação mandibular bi cortical e não é necessário enxerto ósseo. No entanto, a técnica é complexa, difícil e pode causar morbidade significativa envolvendo fratura mandibular e os distúrbios neurossensoriais de anestesia, parestesia e neuralgia ${ }^{29}$.

Dessa forma, os implantes curtos têm ganhado espaço na implantodontia moderna que busca cada vez mais procedimentos menos invasivos e com menor morbidade. O uso de implantes curtos permite o tratamento de pacientes que não são capazes de se submeter a técnicas cirúrgicas complexas por razões médicas, anatômicas ou financeiras. Quando instalados considerando todos os fatores biomecânicos críticos e usando o protocolo clínico estrito os implantes curtos podem ser uma opção bem-sucedida para o tratamento de atrofias ósseas na região mandibular posterior. No entanto, ainda existe uma escassez de dados sobre o sucesso e sobrevivência a longo prazo destes implantes curtos, particularmente no que se refere à carga oclusal, à relação coroa/implante e a perda da qualidade óssea peri-implantar ${ }^{30}$.

\section{CONCLUSÃO}

Em conclusão, os implantes curtos são uma alternativa quando não se tem altura óssea suficiente para instalação de implantes convencionais e que apresenta vantagens e indicações restritas no universo da implantodontia que apresentam bons resultados quando comparados com os implantes de tamanho convencionais. Entretanto, apresenta resultados a curto prazo o que sugere a realização de estudos com longo tempo de acompanhamento que busquem responder sobre a qualidade da técnica nos diversos desafios encontrados na implantodontia.

\section{REFERÊNCIAS}

1. Buser D, Janner SF, Wittneben JG, Bragger U, Ramseier CA, Salvi GE. 10-year survival and success rates of 511 titanium implants with a sandblasted and acid-etched surface: a retrospective study in 303 partially edentulous patients. Clin Implant Dent Relat Res.
2012;14(6):839-51.

2. Zitzmann NU, Scharer P, Marinello CP. Factors influencing the success of GBR. Smoking, timing of implant placement, implant location, bone quality and provisional restoration. $\mathrm{J}$ Clin Periodontol. 1999;26(10):673-82.

3. Srinivasan M, Vazquez L, Rieder P, Moraguez O, Bernard JP, Belser UC. Survival rates of short (6 $\mathrm{mm})$ micro-rough surface implants: a review of literature and meta-analysis. Clin Oral Implants Res. 2014;25(5):539-45.

4. Zhang XM, Shi JY, Gu YX, Qiao SC, Mo JJ, Lai HC. Clinical investigation and patient satisfaction of short implants versus longer implants with osteotome sinus floor elevation in atrophic posterior maxillae: a pilot randomized trial. Clin Implant Dent Relat Res. 2017;19(1):161-66.

5. Lee CT, Chen YW, Starr JR, Chuang SK. Survival analysis of wide dental implant: systematic review and meta-analysis. Clin Oral Implants Res. 2016;27(10):1251-64.

6. Tawil G, Younan R. Clinical evaluation of short, machined-surface implants followed for 12 to 92 months. Int J Oral Maxillofac Implants. 2003; 18(6):894-901.

7. Himmlova L, Dostalova T, Kacovsky A, Konvickova S. Influence of implant length and diameter on stress distribution: a finite element analysis. J Prosthet Dent. 2004;91(1):20-5.

8. Moraes SL, Pellizzer EP, Verri FR, Santiago JF, Jr., Silva JV. Three-dimensional finite element analysis of stress distribution in retention screws of different crown-implant ratios. Comput Methods Biomech Biomed Engin. 2015; 18(7):689-96.

9. Misch CE. Short dental implants: a literature review and rationale for use. Dent Today. 2005; 24(8):64-6, 8.

10. Maeda Y, Satoh T, Sogo M. In vitro differences of stress concentrations for internal and external hex implant-abutment connections: a short communication. J Oral Rehabil. 2006;33(1):75-8.

11. Neves FD, Fones D, Bernardes SR, do Prado CJ, Neto AJ. Short implants--an analysis of longitudinal studies. Int $\mathrm{J}$ Oral Maxillofac Implants. 2006;21(1):86-93.

12. Renouard F, Nisand D. Impact of implant length and diameter on survival rates. Clin Oral Implants Res. 2006;17(Suppl 2):35-51.

13. Telleman G, Raghoebar GM, Vissink A, den Hartog L, Huddleston Slater JJ, Meijer HJ. A systematic review of the prognosis of short $(<10$ $\mathrm{mm}$ ) dental implants placed in the partially edentulous patient. J Clin Periodontol. 2011; 38(7):667-76.

14. Schincaglia GP, Thoma DS, Haas R, Tutak M, Garcia A, Taylor TD, et al. Randomized controlled multicenter study comparing short 
dental implants $(6 \mathrm{~mm})$ versus longer dental implants $(11-15 \mathrm{~mm})$ in combination with sinus floor elevation procedures. Part 2: clinical and radiographic outcomes at 1 year of loading. J Clin Periodontol. 2015;42(11):1042-51.

15. Bechara S, Kubilius R, Veronesi G, Pires JT, Shibli JA, Mangano FG. Short (6-mm) dental implants versus sinus floor elevation and placement of longer $(>/=10-\mathrm{mm})$ dental implants: a randomized controlled trial with a 3-year follow-up. Clin Oral Implants Res. 2017; 28(9):1097-1107.

16. Sun C, Zhao J, Jianghao C, Hong T. Effect of Heavy Smoking on Dental Implants Placed in Male Patients Posterior Mandibles: A Prospective Clinical Study. J Oral Implantol. 2016; 42(6):477-83.

17. Al Amri MD, Abduljabbar TS, Al-Johany SS, Al Rifaiy MQ, Alfarraj Aldosari AM, Al-Kheraif AA. Comparison of clinical and radiographic parameters around short ( 6 to $8 \mathrm{~mm}$ in length) and long (11 $\mathrm{mm}$ in length) dental implants placed in patients with and without type 2 diabetes mellitus: 3 -year follow-up results. Clin Oral Implants Res. 2017;28(10):1182-87.

18. Nkenke E, Stelzle F. Clinical outcomes of sinus floor augmentation for implant placement using autogenous bone or bone substitutes: a systematic review. Clin Oral Implants Res. 2009; 20(Suppl 4):124-33.

19. Silva LD, de Lima VN, Faverani LP, de Mendonça MR, Okamoto R, Pellizzer EP. Maxillary sinus lift surgery-with or without graft material? A systematic review. Int $\mathrm{J}$ Oral Maxillofac Surg. 2016;45(12):1570-76.

20. Barone A, Santini S, Sbordone L, Crespi R, Covani U. A clinical study of the outcomes and complications associated with maxillary sinus augmentation. Int $\mathbf{J}$ Oral Maxillofac Implants. 2006;21(1):81-5.

21. Atieh MA, Zadeh H, Stanford CM, Cooper LF. Survival of short dental implants for treatment of posterior partial edentulism: a systematic review. Int J Oral Maxillofac Implants. 2012; 27(6):1323-31.

22. Tan WC, Lang NP, Zwahlen M, Pjetursson BE. A systematic review of the success of sinus floor elevation and survival of implants inserted in combination with sinus floor elevation. Part II: transalveolar technique. J Clin Periodontol. 2008; 35(8 Suppl):241-54.

23. French D, Larjava H, Ofec R. Retrospective cohort study of 4591 Straumann implants in private practice setting, with up to 10-year followup. Part 1: multivariate survival analysis. Clin Oral Implants Res. 2015;26(11):1345-54.

24. Perelli M, Abundo R, Corrente G, Saccone C. Short (5 and $7 \mathrm{~mm}$ long) porous implants in the posterior atrophic maxilla: a 5-year report of a prospective single-cohort study. Eur J Oral Implantol. 2012;5(3):265-72.

25. Morrison A, Chiarot M, Kirby S. Mental nerve function after inferior alveolar nerve transposition for placement of dental implants. J Can Dent Assoc. 2002;68(1):46-50.

26. Chiapasco M, Zaniboni M, Rimondini L. Autogenous onlay bone grafts vs. alveolar distraction osteogenesis for the correction of vertically deficient edentulous ridges: a 2-4-year prospective study on humans. Clin Oral Implants Res. 2007;18(4):432-40.

27. Berglundh T, Persson L, Klinge B. A systematic review of the incidence of biological and technical complications in implant dentistry reported in prospective longitudinal studies of at least 5 years. J Clin Periodontol. 2002;29(Suppl 3):197-212.

28. Chrcanovic BR, Custodio AL. Inferior alveolar nerve lateral transposition. Oral Maxillofac Surg. 2009;13(4):213-19.

29. Abayev B, Juodzbalys G. Inferior Alveolar Nerve Lateralization and Transposition for Dental Implant Placement. Part II: a Systematic Review of Neurosensory Complications. J Oral Maxillofac Res. 2015;6(1):e3.

30. Jain N, Gulati M, Garg M, Pathak C. Short Implants: New Horizon in Implant Dentistry. J Clin Diagn Res. 2016;10(9):ZE14-7.

\section{CONFLITO DE INTERESSES}

Os autores declaram não haver conflitos de interesse.

\section{AUTOR PARA CORRESPONDENCIA}

Guilherme André Del'Arco Ramires

guilherme_adr@hotmail.com

Submetido em 10/04/2018

Aceito em 27/06/2018 